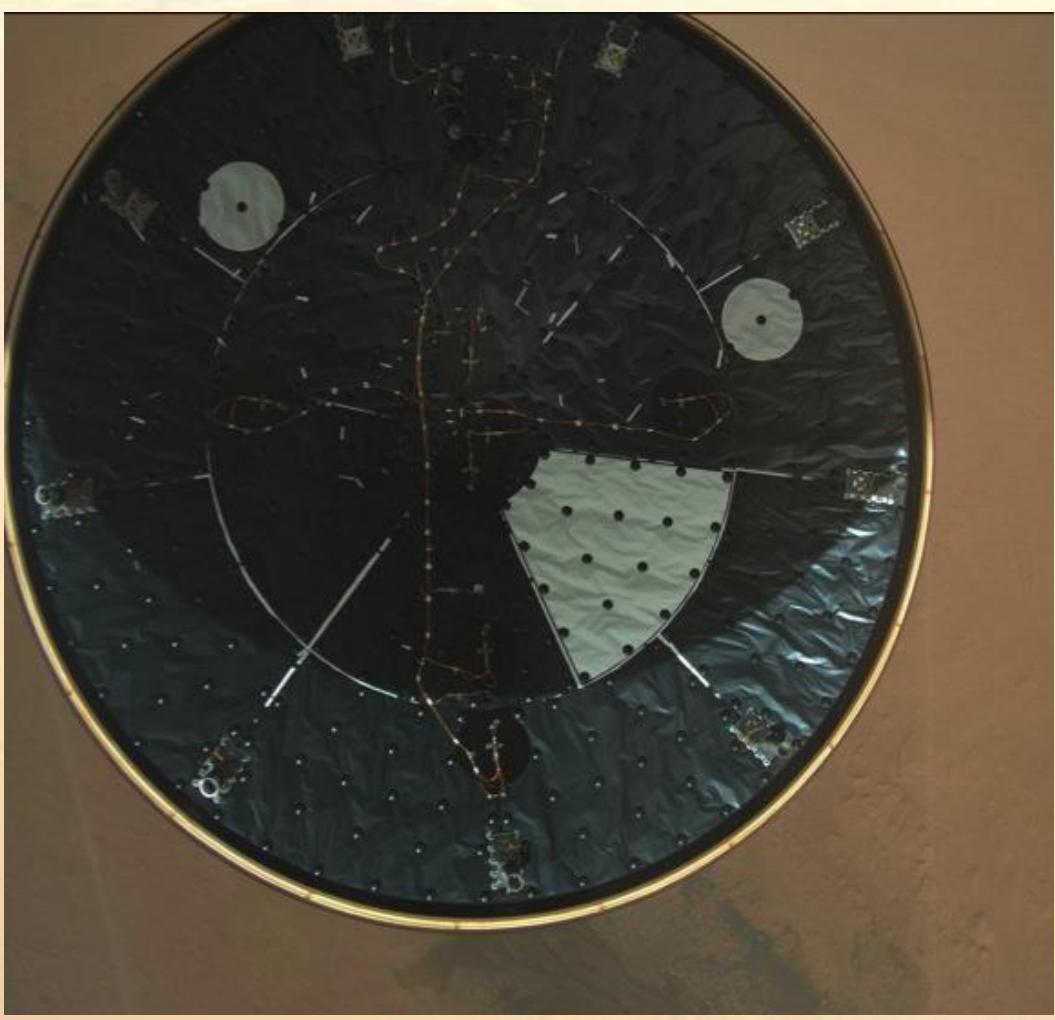

\title{
Characterization of a Radiometer Window for Mars Aftbody Heating Including Ablation Product Deposition Using a Miniature Arc Jet
}

Ruth A. Miller, Stanford University

Chun Tang, Mark S. McGlaughlin, Todd R. White, NASA Ames Research Center Thanh S. Ho, Megan MacDonald, Jacobs Technology at NASA Ames Research Center Brett A. Cruden, AMA Inc. at NASA Ames Research Center 


\section{Background}

- Mars Entry Descent and Landing Instrumentation 2 (MEDLI2)

- Characterize aerodynamic, aerothermodynamic, and TPS performance

- Expands off MEDLI (Mars Science Laboratory, 2012)

- Pressure Transducers, Thermal Plugs, Heat Flux Sensors, Radiometer

\section{MEDLI2 (Mars 2020)}

Thermal Instrumentation

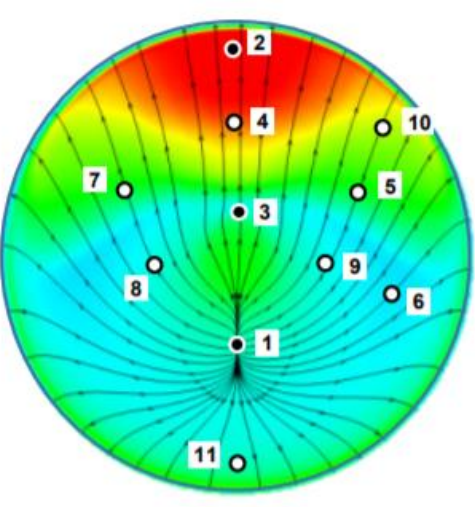

- PICA Aerothermal Plug

- PICA Thermal Response Plug Heatshield

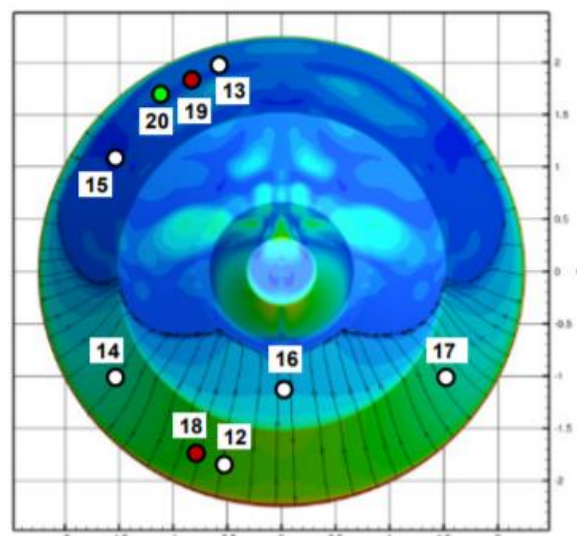

O SLA-561V Aerothermal Plug - Heatflux Sensor O Radiometer Backshell

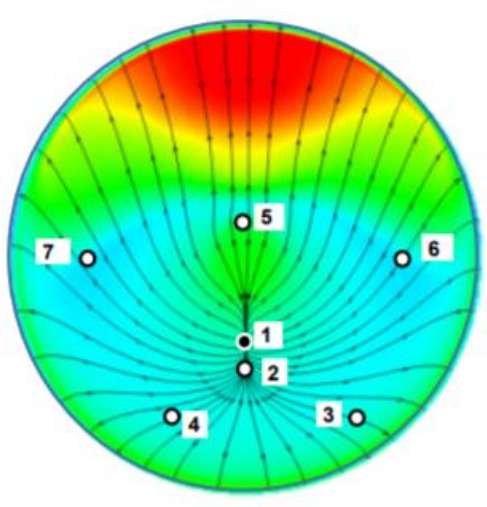

- Supersonic Pressure - Hypersonic Pressure Heatshield

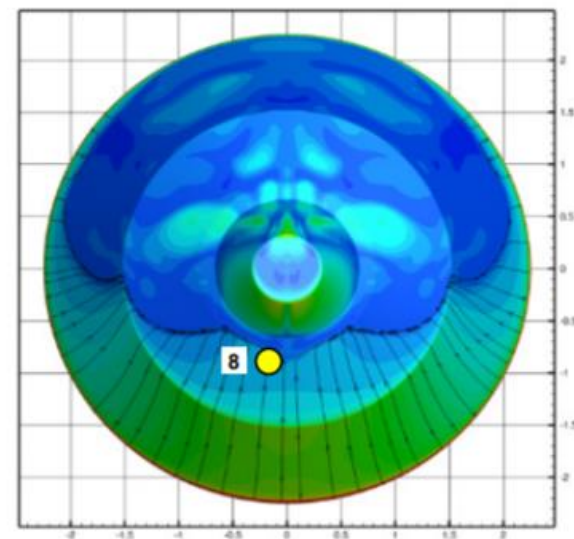

Backshell Pressure Backshell

Figure Credit: H. H. Hwang et al., 2016. 


\section{MEDLI2 Radiometer}

- MEDLI2 radiometer consists of a heat flux sensor behind a sapphire window

- Measurement range: $0-15 \mathrm{~W} / \mathrm{cm}^{2}$

MEDLI2 Radiometer

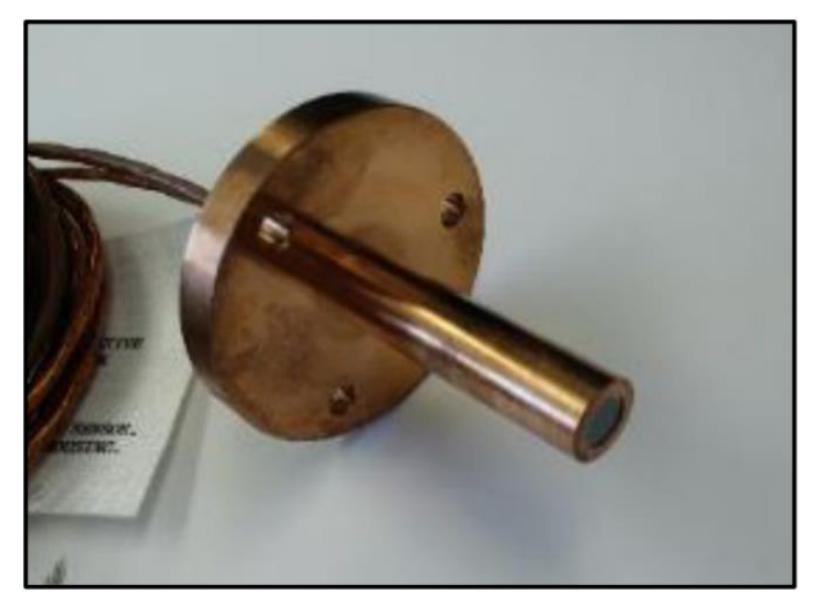

- Expected backshell heating for Mars 2020:

- Radiative: $~ 4.5 \mathrm{~W} / \mathrm{cm}^{2}$

- Convective: $~ 1 \mathrm{~W} / \mathrm{cm}^{2}$
- Spectral radiance for MSL entry from Electric Arc Shock Tube (EAST) experiments show peak radiance around $2.7 \mu \mathrm{m}$ and $4.3 \mu \mathrm{m}$ due to $\mathrm{CO}_{2}$

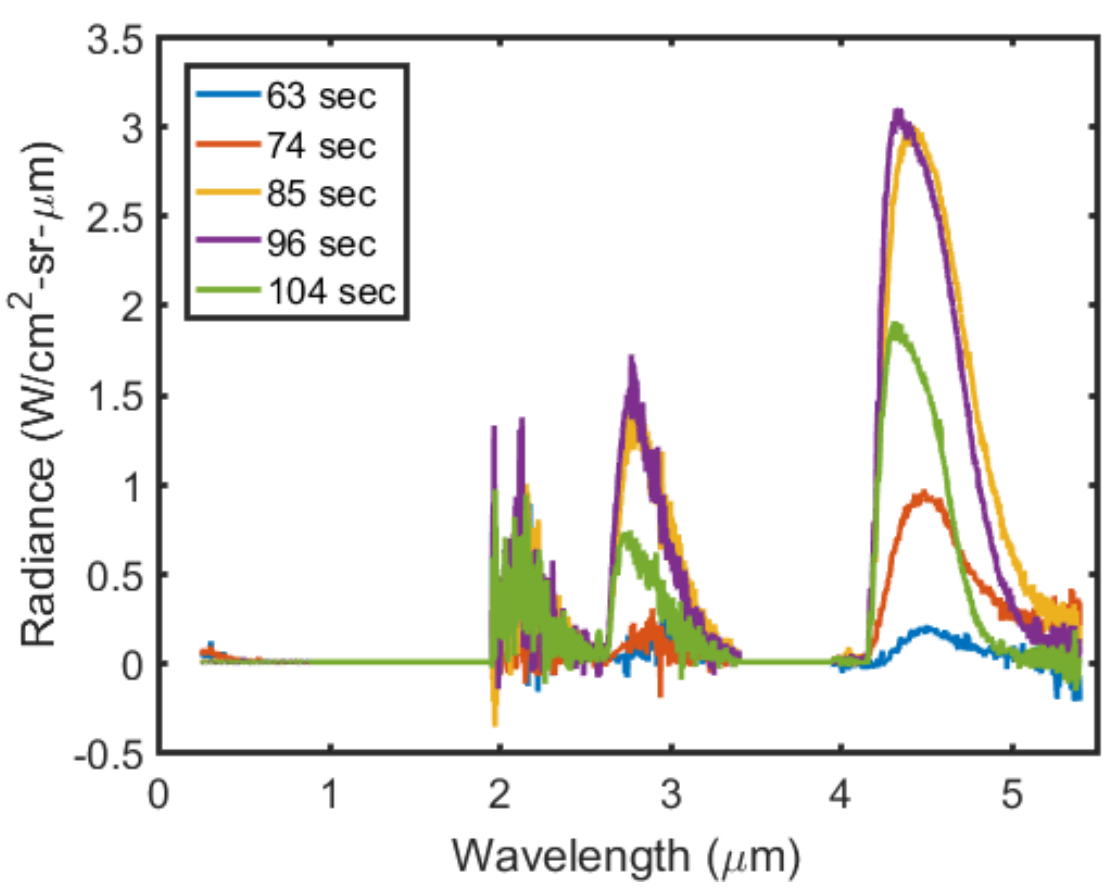

Figure Credit: B. A. Cruden et al., 2015. 


\section{Radiometer Characterization}

- Radiometer output

$V_{\text {rad }} \propto \int T(\lambda) A(\lambda) L(\lambda) d \lambda$

$L(\lambda)$ : Spectral radiance of source (calibration or shock layer)

$T(\lambda)$ : Spectral transmission of window

$A(\lambda)$ : Spectral absorbance of heat flux sensor

- Radiometer output is not spectral, however

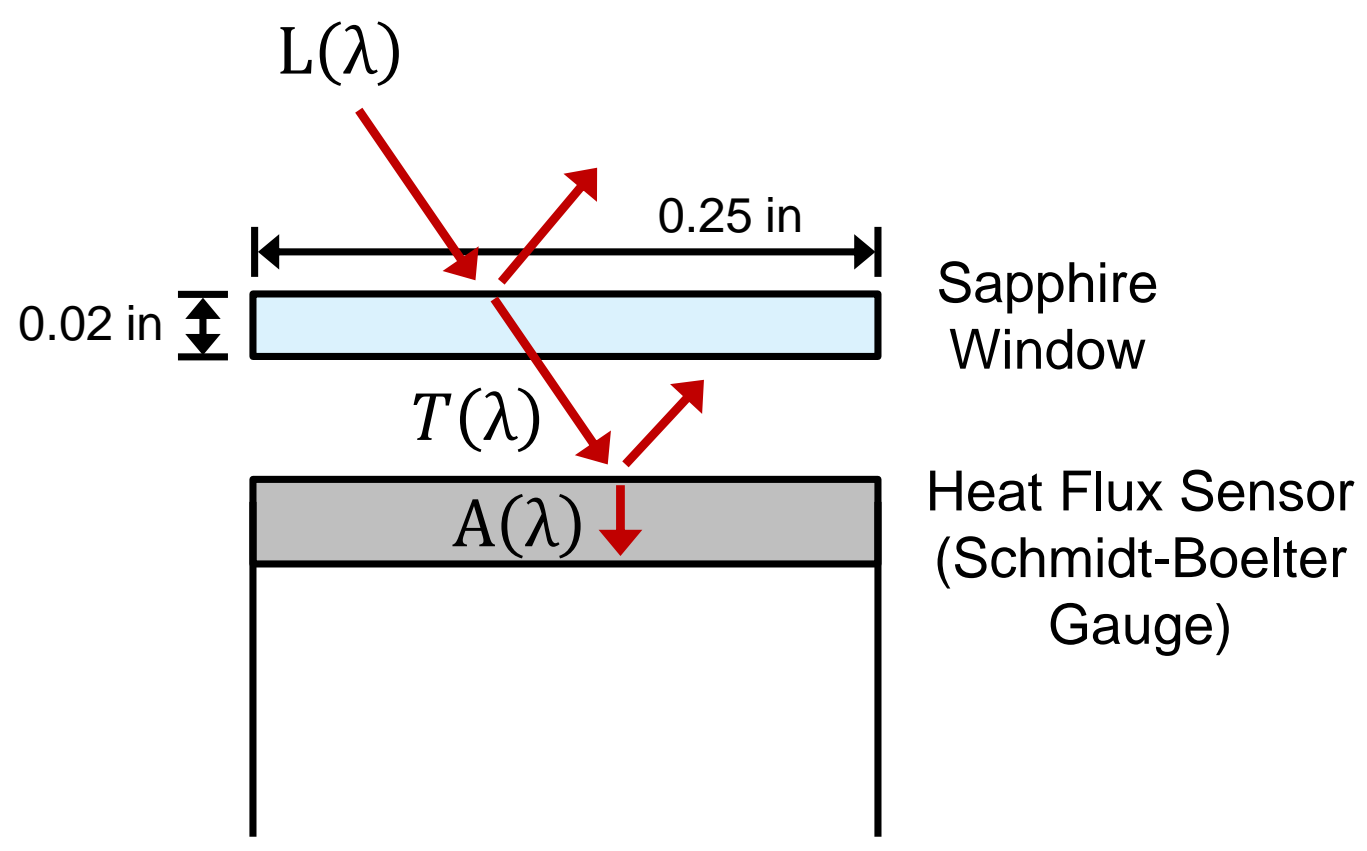
characterization will be done spectrally 


\section{Transmission and Absorbance}

Sapphire Window

- Transmission of $\sim 88 \%$ up to $5.5 \mu \mathrm{m}$

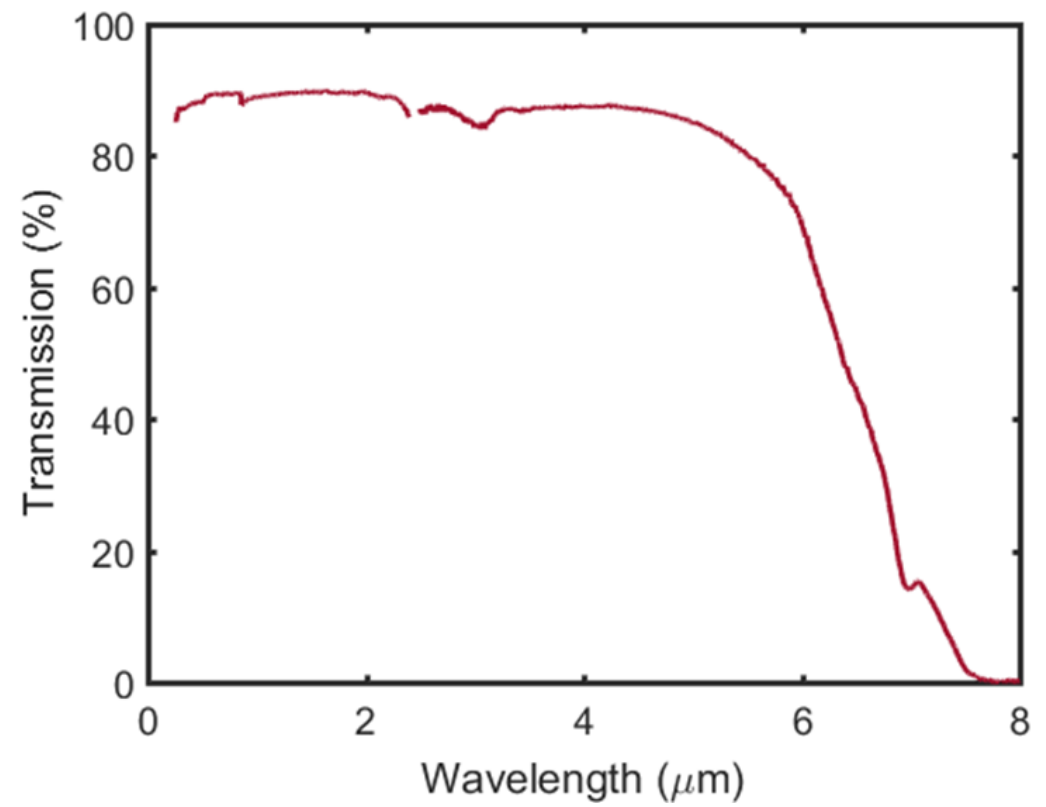

$\underline{\text { Heat Flux Sensor }}$

- Absorbance of 95\%
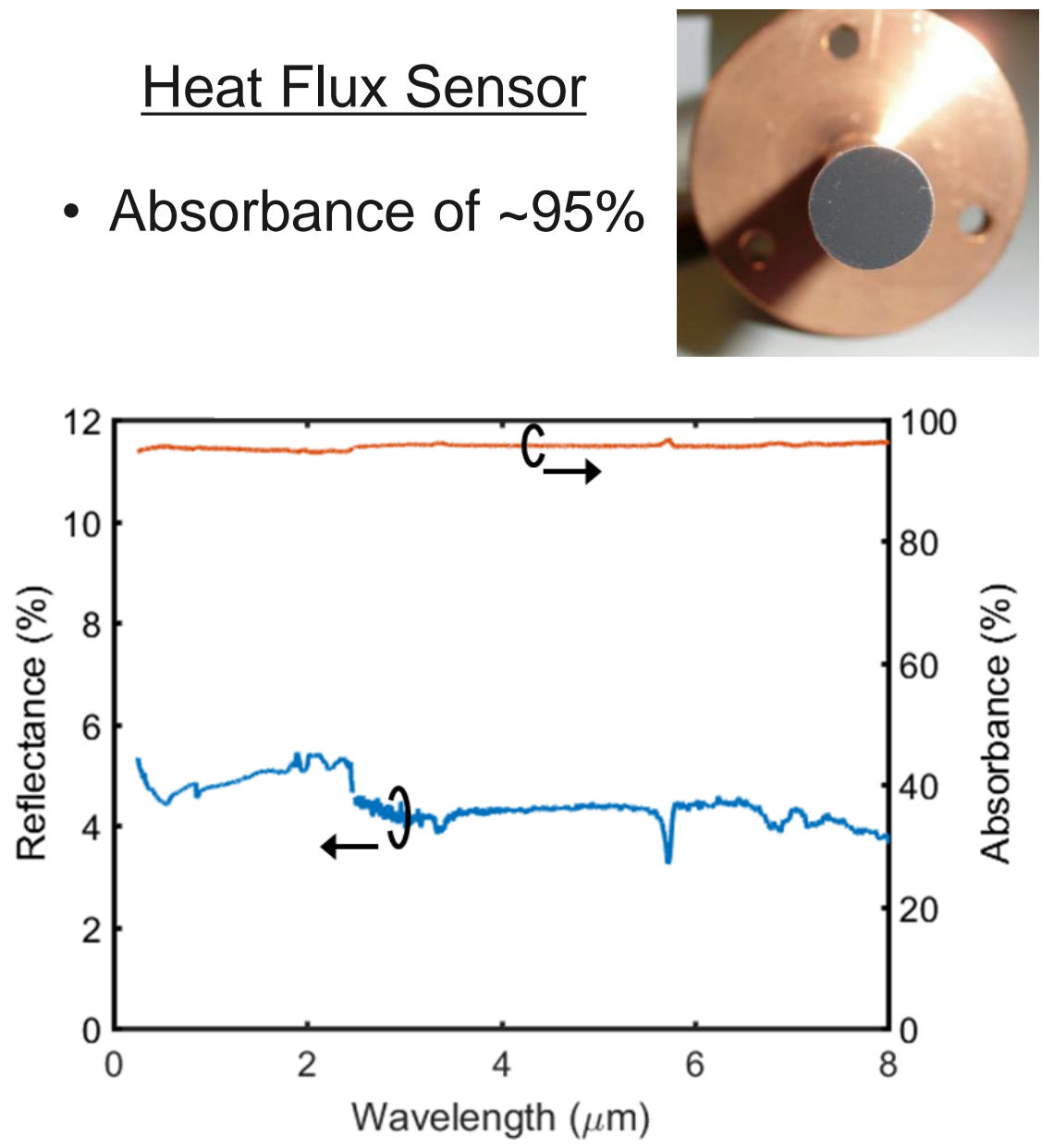


\section{Measured Radiation}

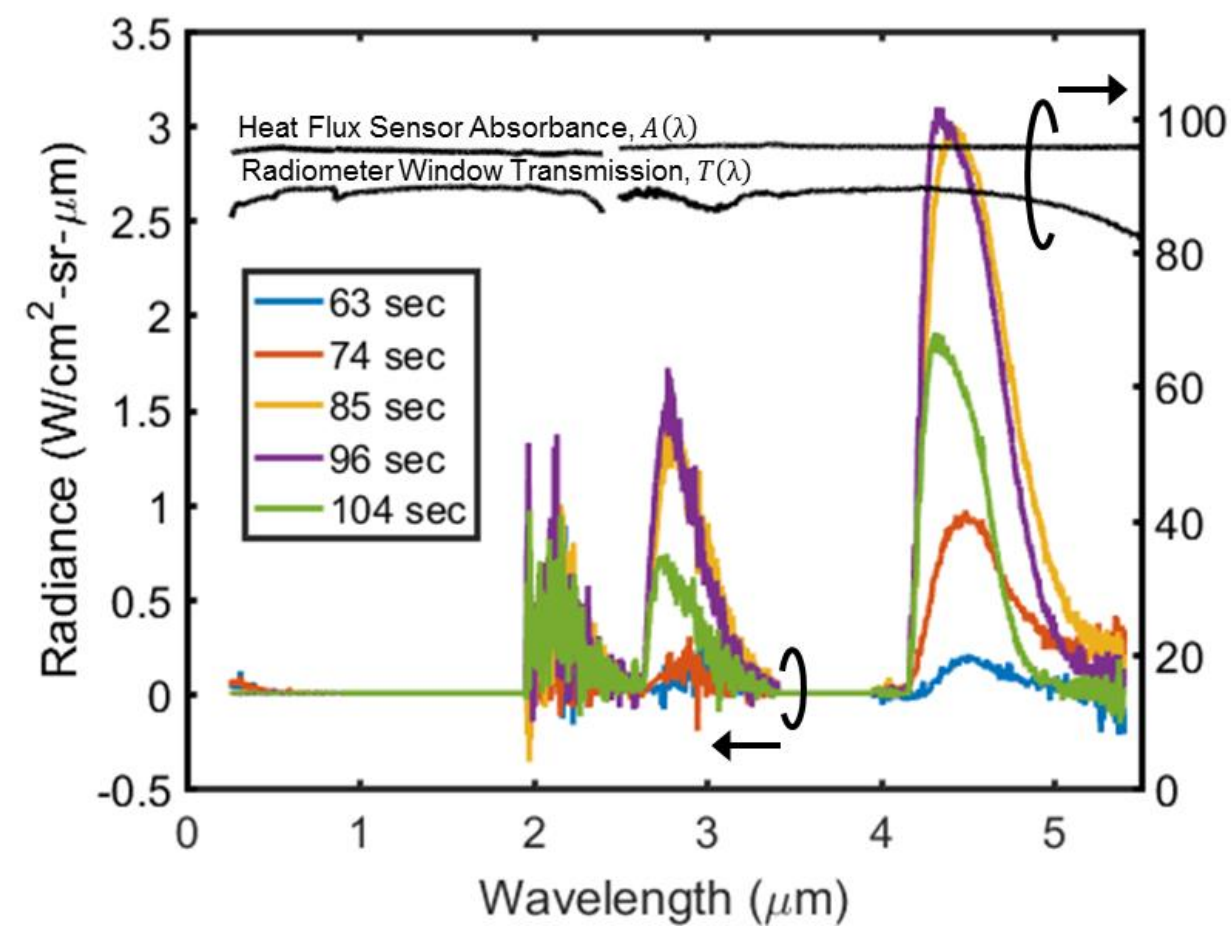

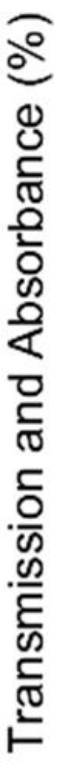




\section{Measured Radiation}

Percent Signal Loss:

$$
S_{\text {loss }}=\left(1-\frac{\int T(\lambda) A(\lambda) L(\lambda) d \lambda}{T_{c a l} A_{c a l} \int L(\lambda) d \lambda}\right) \times 100
$$

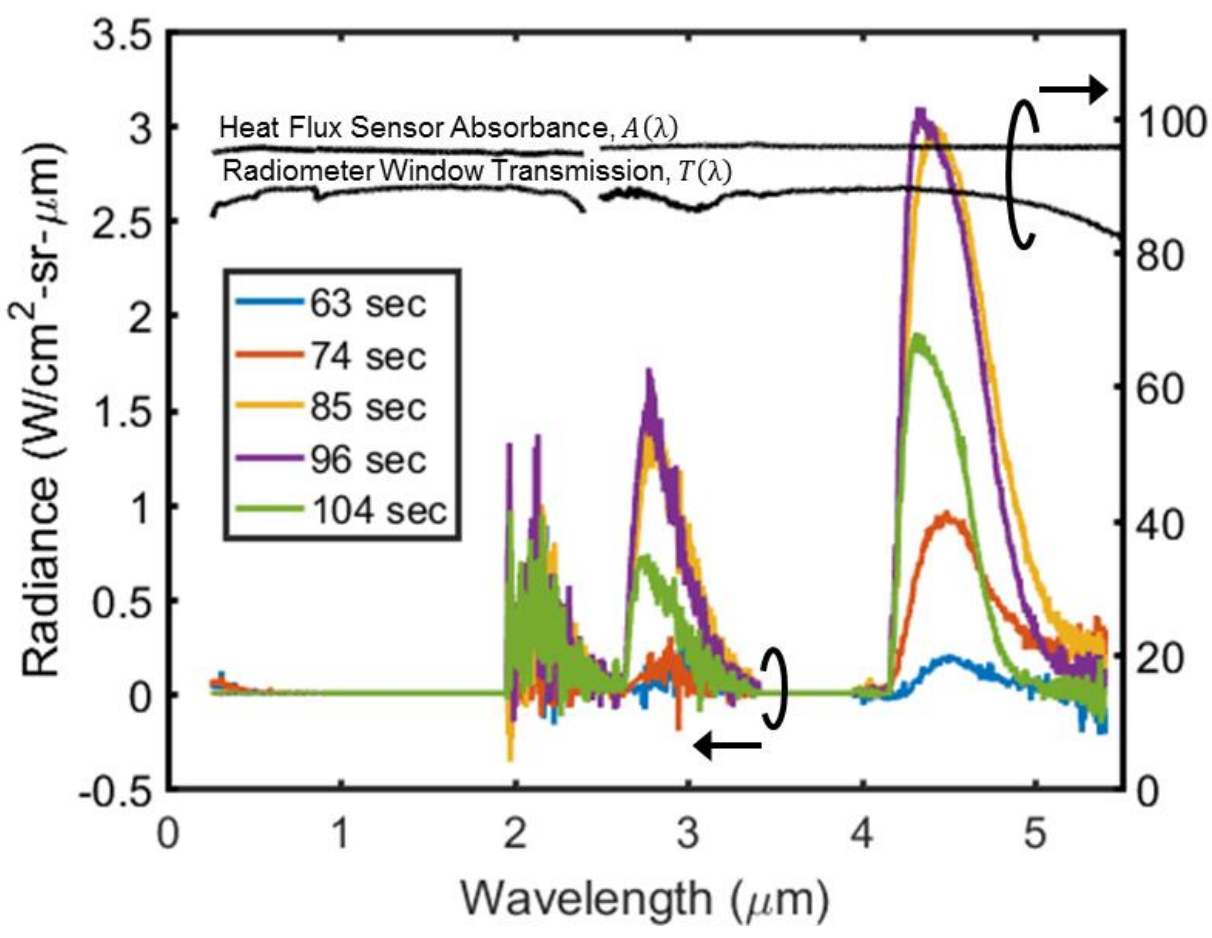

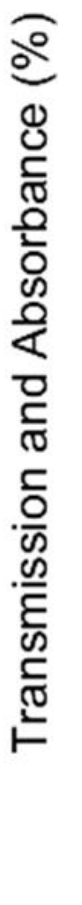




\section{Measured Radiation}

Percent Signal Loss:

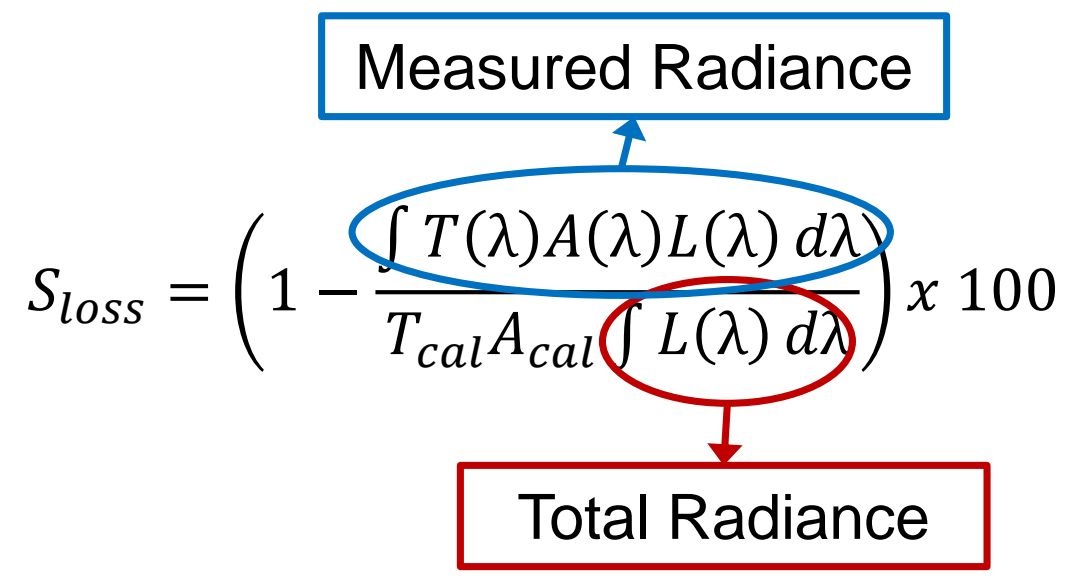

$>$ Measure of unaccounted for radiation due to variation in wavelength sensitivity

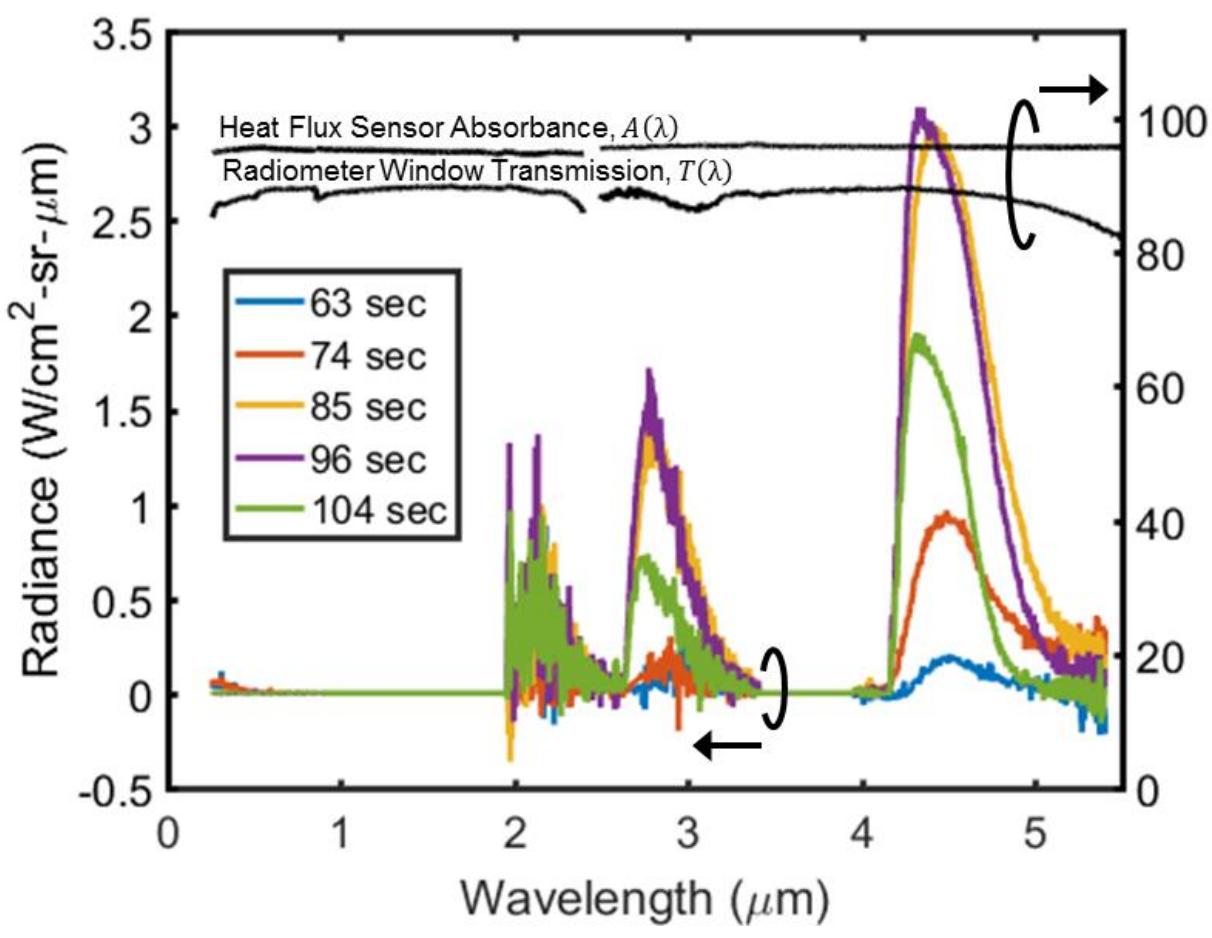

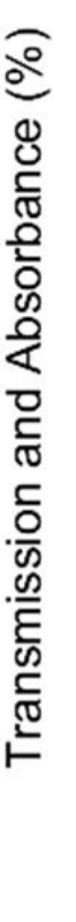




\section{Measured Radiation}

Percent Signal Loss:

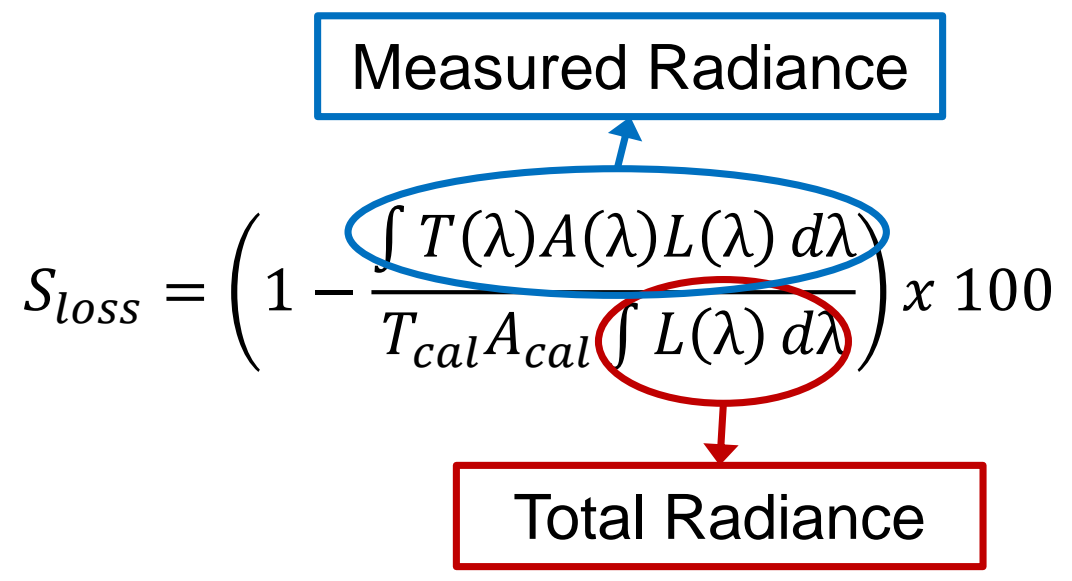

$>$ Measure of unaccounted for radiation due to variation in wavelength sensitivity

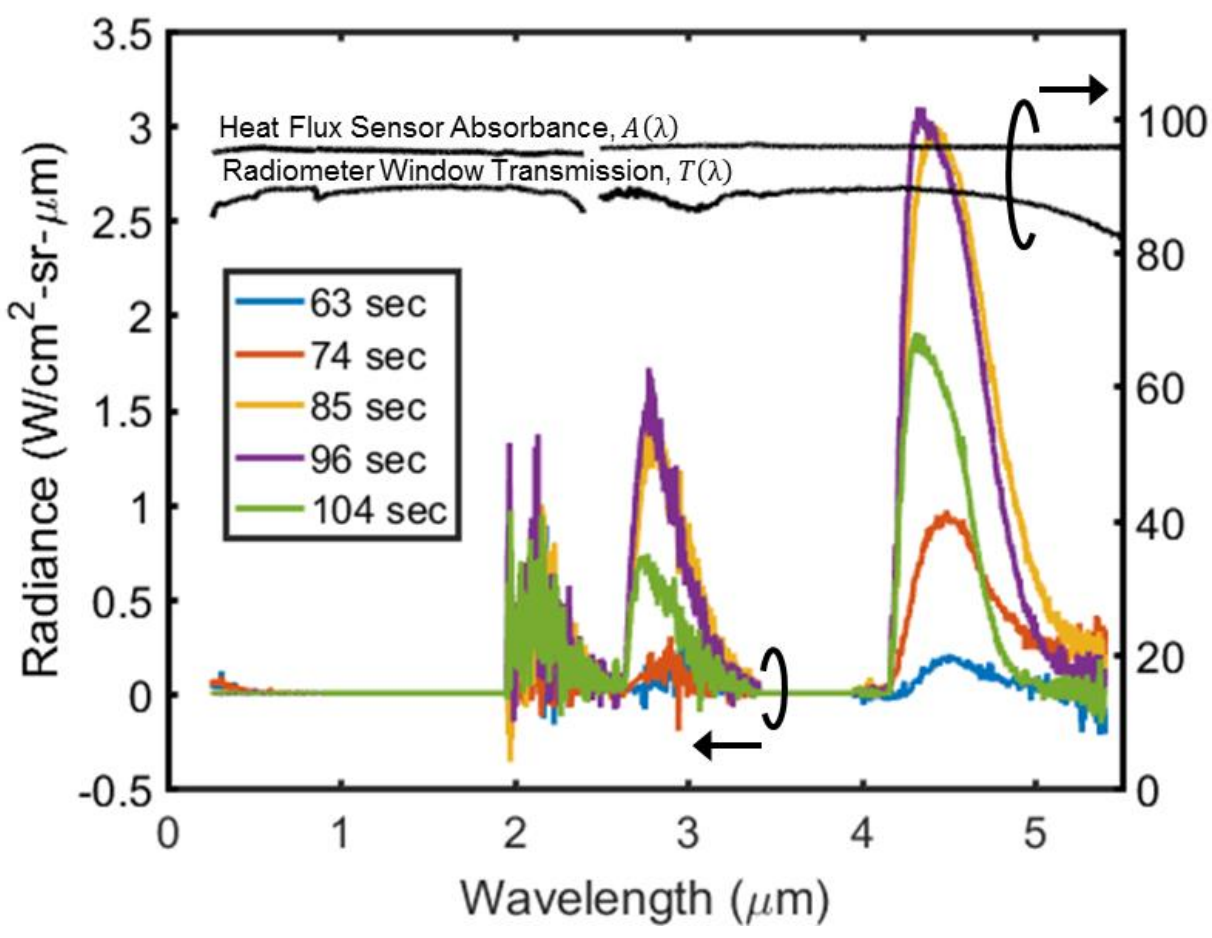

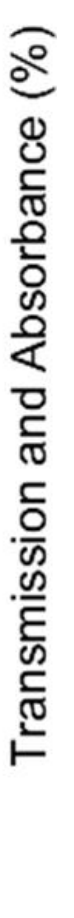

- Assuming radiometer calibration corrects for 1 - $2 \mu \mathrm{m} \rightarrow T_{\text {cal }} A_{\text {cal }}=85 \%$

$>S_{\text {loss }}=1.8 \%$ ! 


\section{Thermal Protection System (TPS) Ablation}

- Radiometer will be embedded in backshell TPS

- TPS ablation products from heatshield or backshell could be deposited on the window
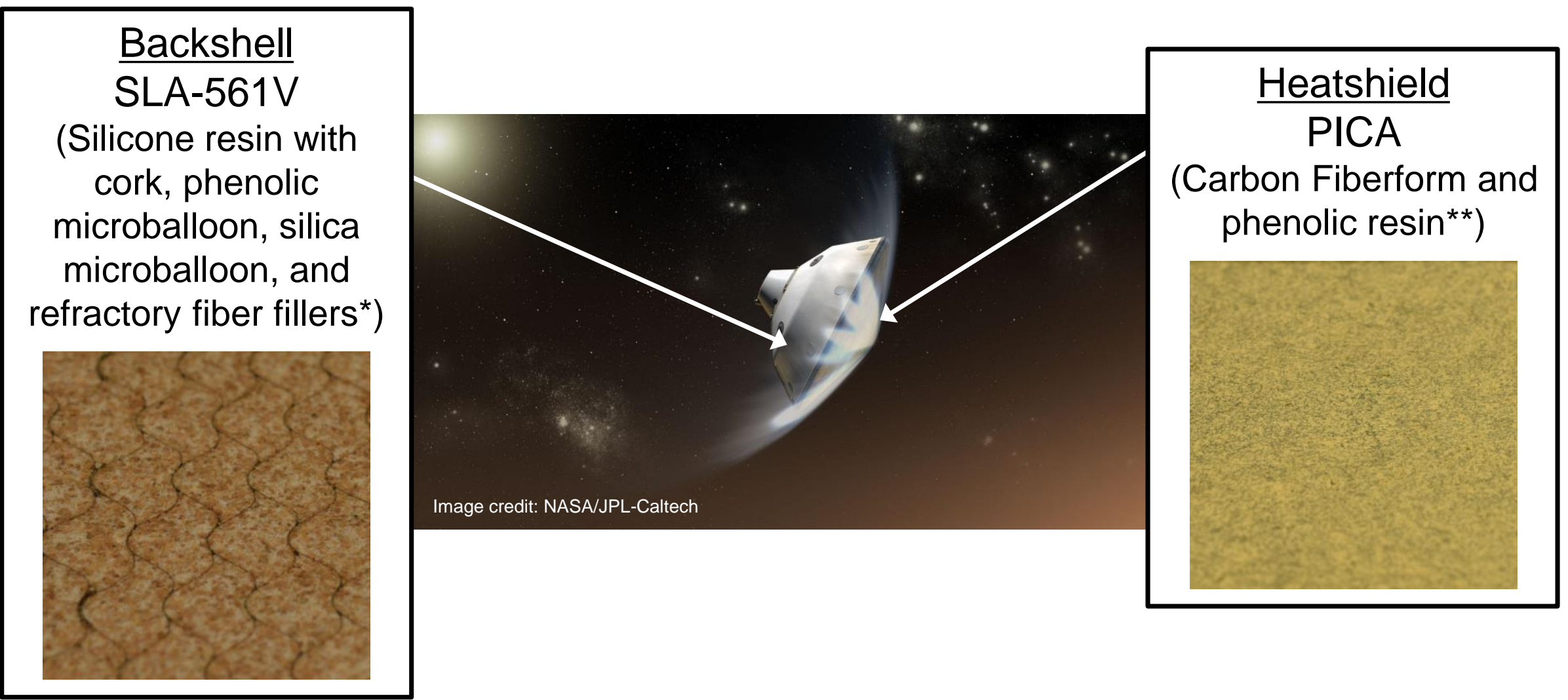


\section{Miniature Arc Jet (mARC) Facility}

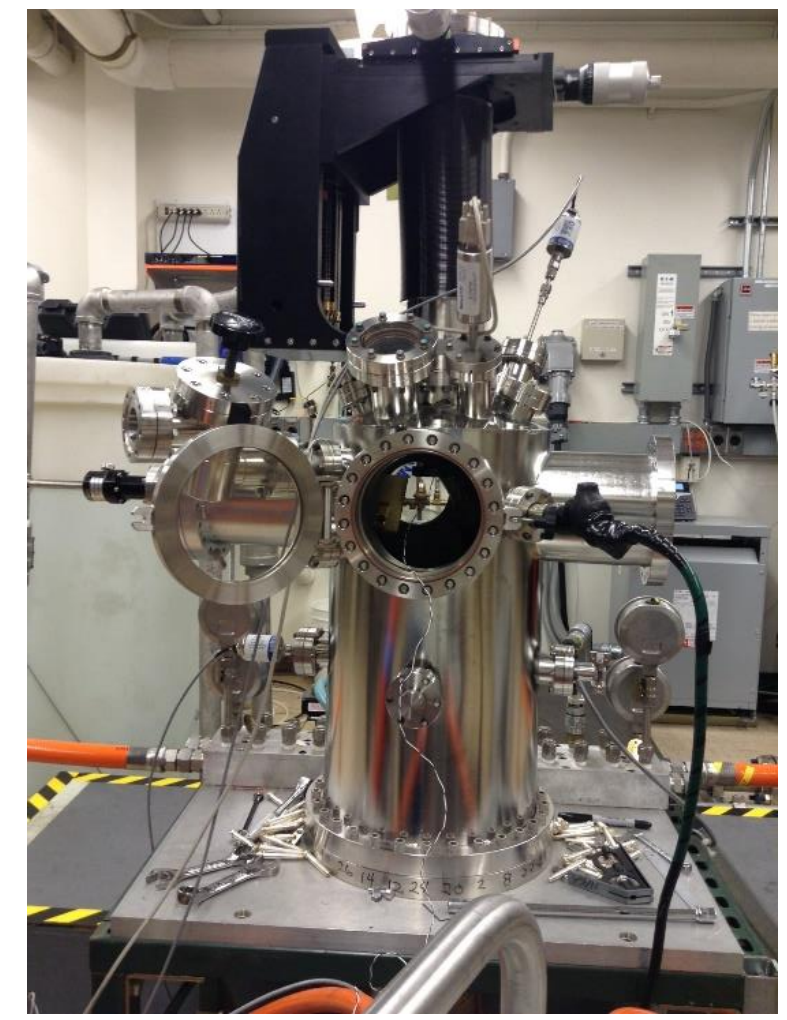

- mARC previously

mARC Flow characterized in air*

- Heat fluxes $>1000 \mathrm{~W} / \mathrm{cm}^{2}$

- Expected Mars 2020 heat flux:

- Heatshield: $150 \mathrm{~W} / \mathrm{cm}^{2}$

- Backshell: $5 \mathrm{~W} / \mathrm{cm}^{2}$

- Characterize mARC in Mars flight relevant environment $\left(90 \% \mathrm{CO}_{2}\right.$, $10 \% \mathrm{~N}_{2}$ )

Pitot Probe at $20 \mathrm{~mm}$ from Nozzle

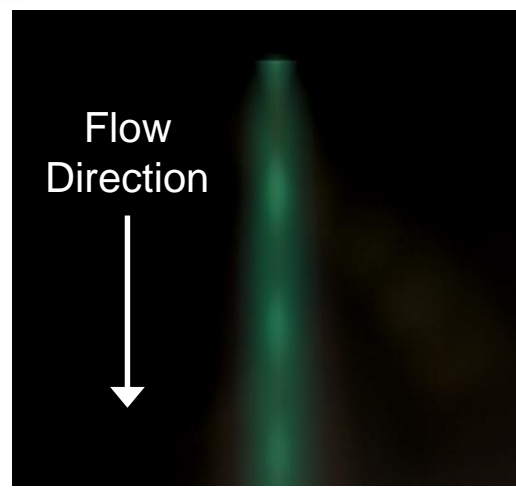

Gardon Gauge at $2 \mathrm{~mm}$ from Nozzle

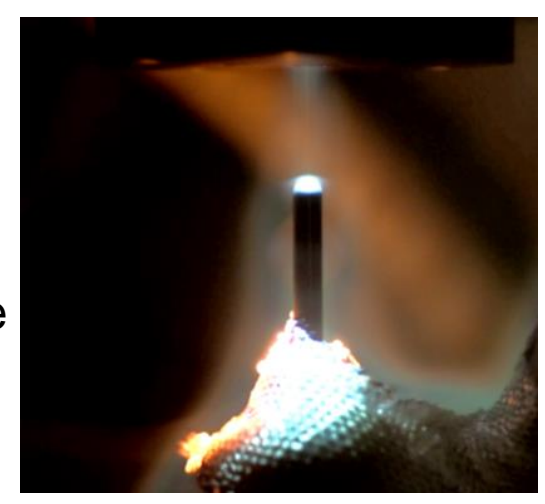




\section{mARC Characterization Results}

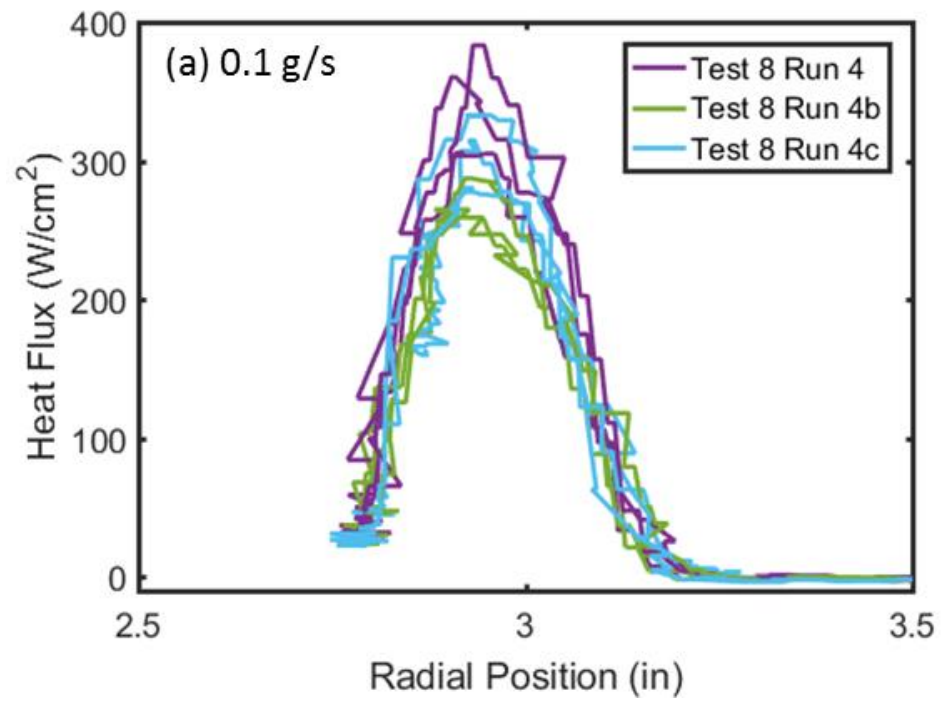

Flow rate of $0.1 \mathrm{~g} / \mathrm{s}$

$\rightarrow$ Centerline heat flux: $250-400 \mathrm{~W} / \mathrm{cm}^{2}$

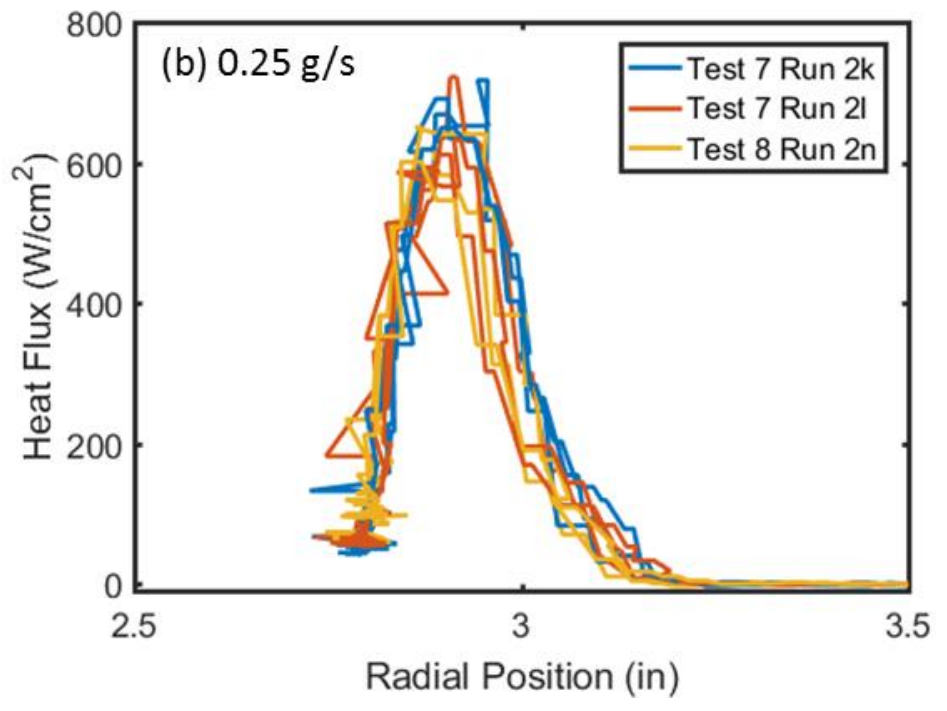

Flow rate of $0.25 \mathrm{~g} / \mathrm{s}$

$\rightarrow$ Centerline heat flux: $550-700 \mathrm{~W} / \mathrm{cm}^{2}$

Test Conditions:

- $90 \% \mathrm{CO}_{2}, 10 \% \mathrm{~N}_{2}$ (by mass)

- Arc Current: $40 \mathrm{~A}$

- Distance From Nozzle: 20 mm

See paper for detailed mARC characterization results. 


\section{Test Matrix}

Test Conditions:

- $90 \% \mathrm{CO}_{2}, 10 \% \mathrm{~N}_{2}$ (by mass)

- Mass Flow Rate: $0.1 \mathrm{~g} / \mathrm{s}$

- Arc Current: $40 \mathrm{~A}$

- Distance From Nozzle: $20 \mathrm{~mm}$

- Model Inclination Angle: $45^{\circ}$

Impingement

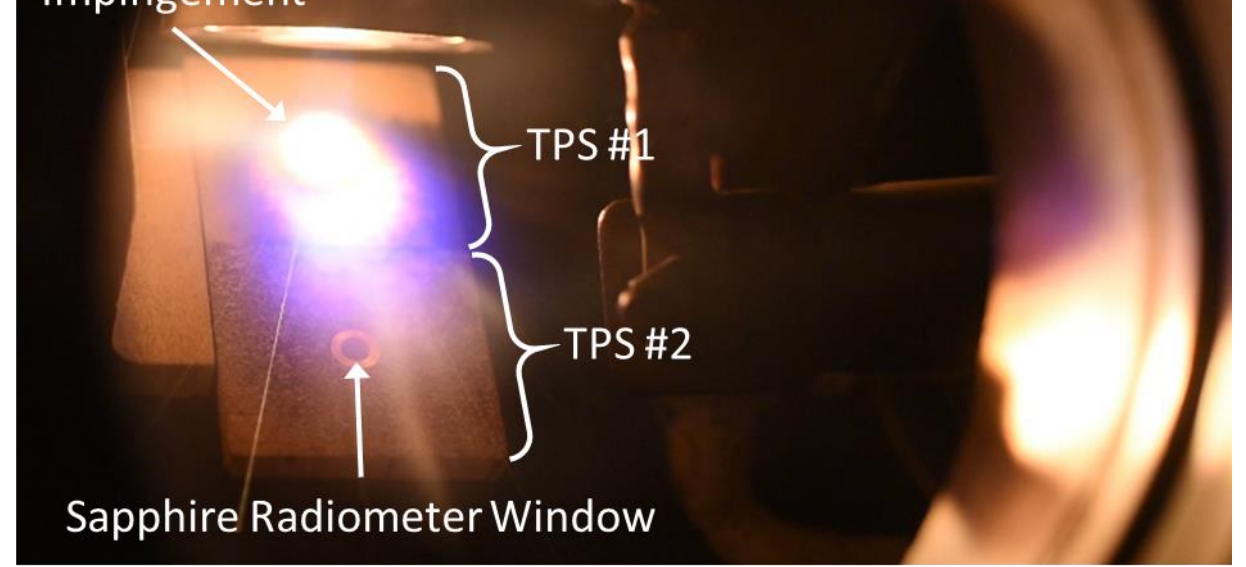

\begin{tabular}{ccccc}
\hline $\begin{array}{c}\text { Run } \\
\text { Number }\end{array}$ & $\begin{array}{c}\text { Window } \\
\text { Number }\end{array}$ & $\begin{array}{c}\text { Duration } \\
(\mathbf{s e c})\end{array}$ & TPS \#1/TPS \#2 & $\begin{array}{c}\text { Copper } \\
\text { Slug? }\end{array}$ \\
\hline 1 & 1 & 55 & PICA/PICA & Yes \\
2 & 4 & 40 & PICA/PICA & No \\
3 & 3 & 30 & PICA/SLA & Yes \\
\hline 4 & 6 & 30 & PICA/SLA & No \\
\hline 5 & 2 & 30 & SLA/SLA & Yes \\
6 & 5 & 30 & SLA/SLA & No \\
\hline
\end{tabular}




\section{Radiometer Windows}

PICA/PICA, with Copper Slug, Window 1

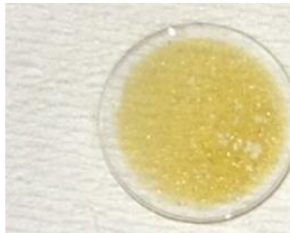

PICA/PICA with Copper Slug Window 1

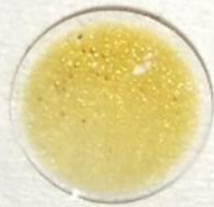

PICA/PICA without Copper Slug Window 4

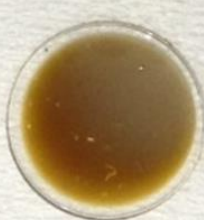

SLA/SLA with Copper Slug Window 2

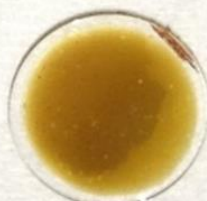

SLA/SLA without Copper Slug Window 5

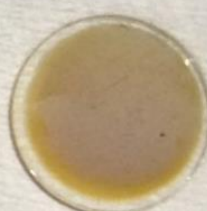

PICA/SLA with Copper Slug Window 3

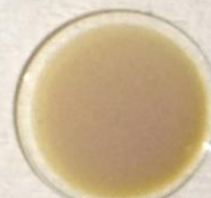

PICA/SLA without Copper Slug Window 6
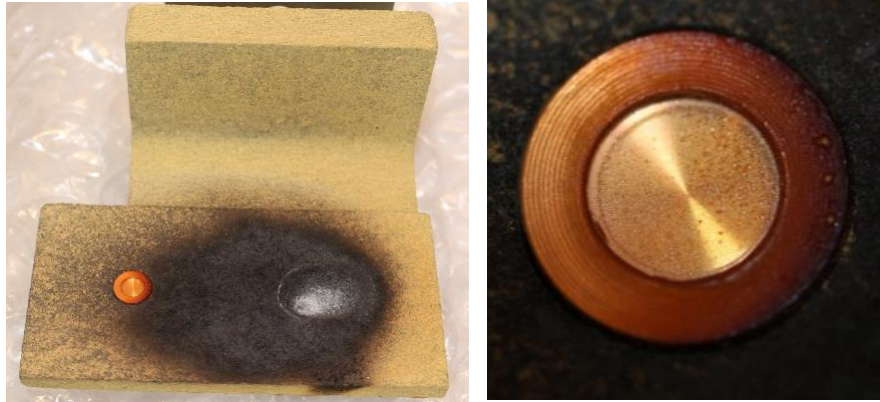

PICA/SLA, with Copper Slug, Window 3
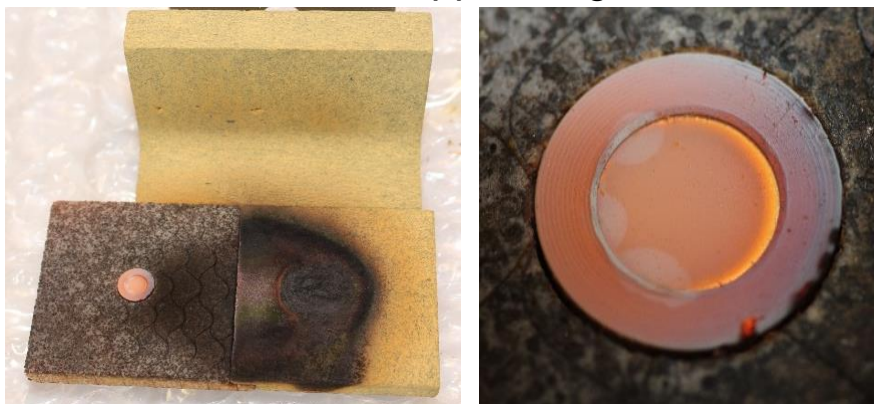

SLA/SLA, with Copper Slug, Window 2
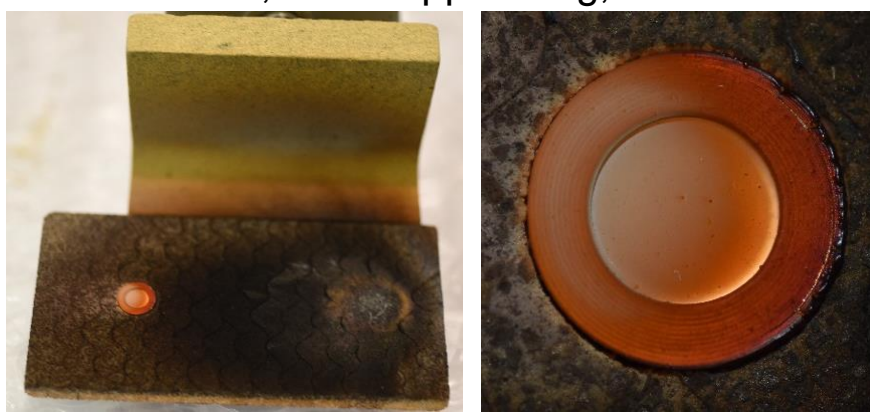


\section{Radiometer Window Transmission Post-mARC Test}

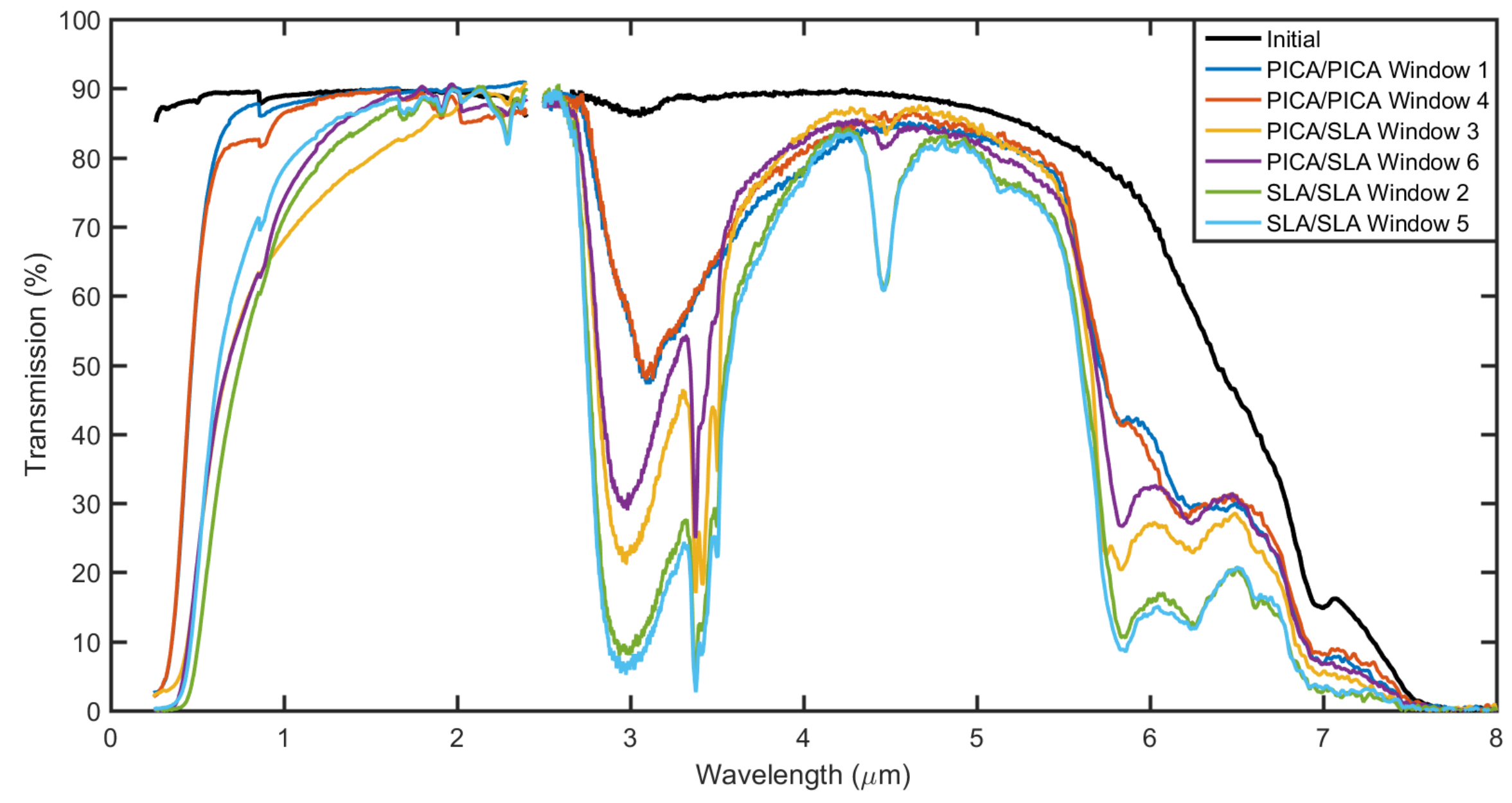




\section{Radiometer Window Transmission Post-mARC Test}
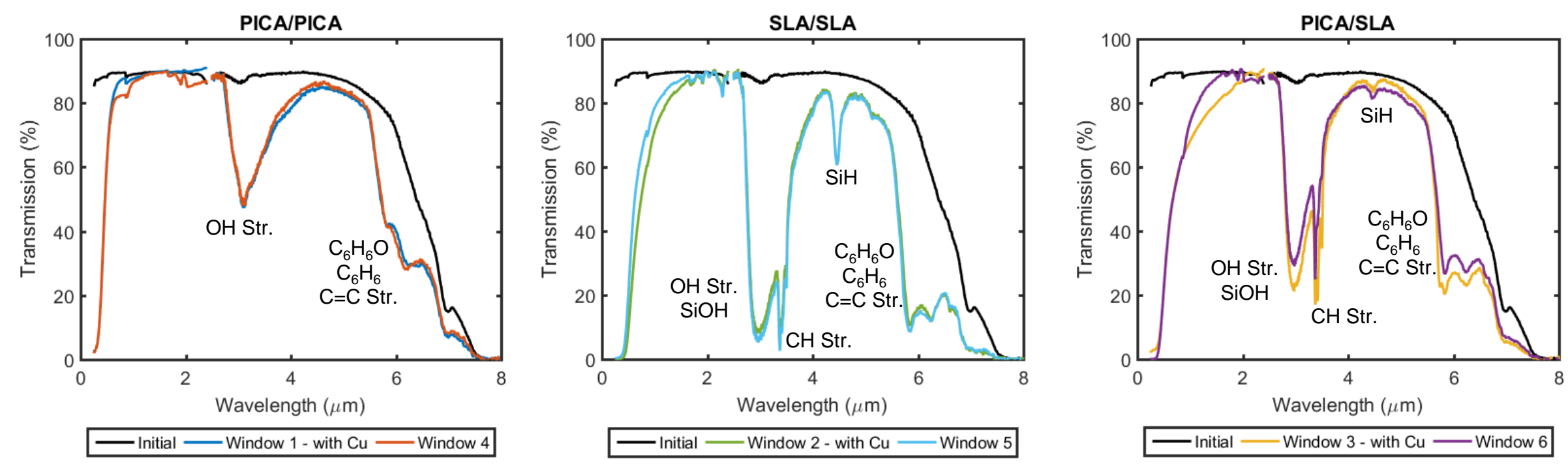


\section{Comparison to EAST MSL Data}
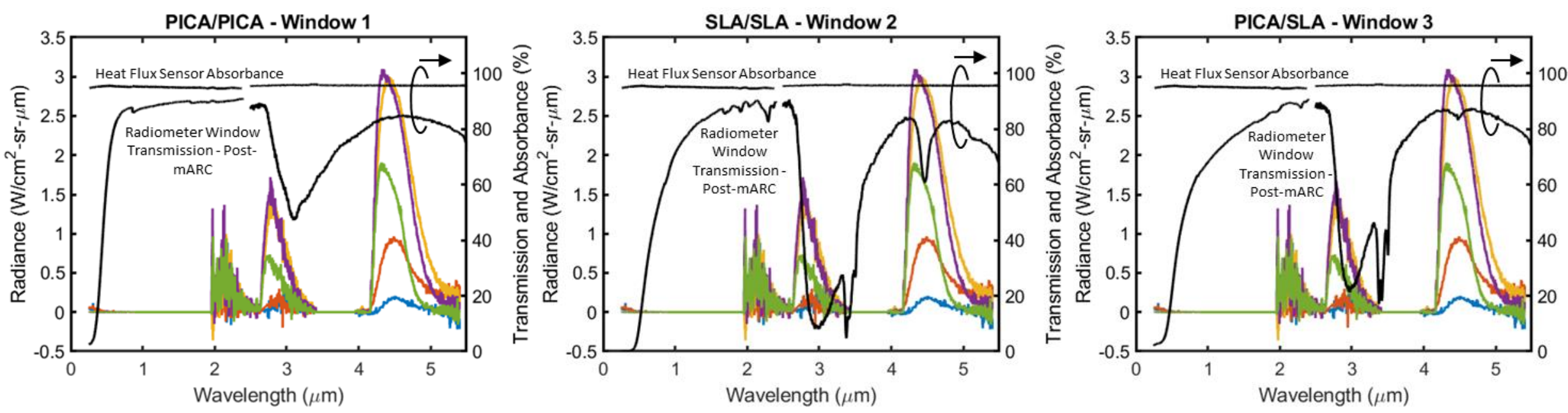


\section{Percent Signal Loss}

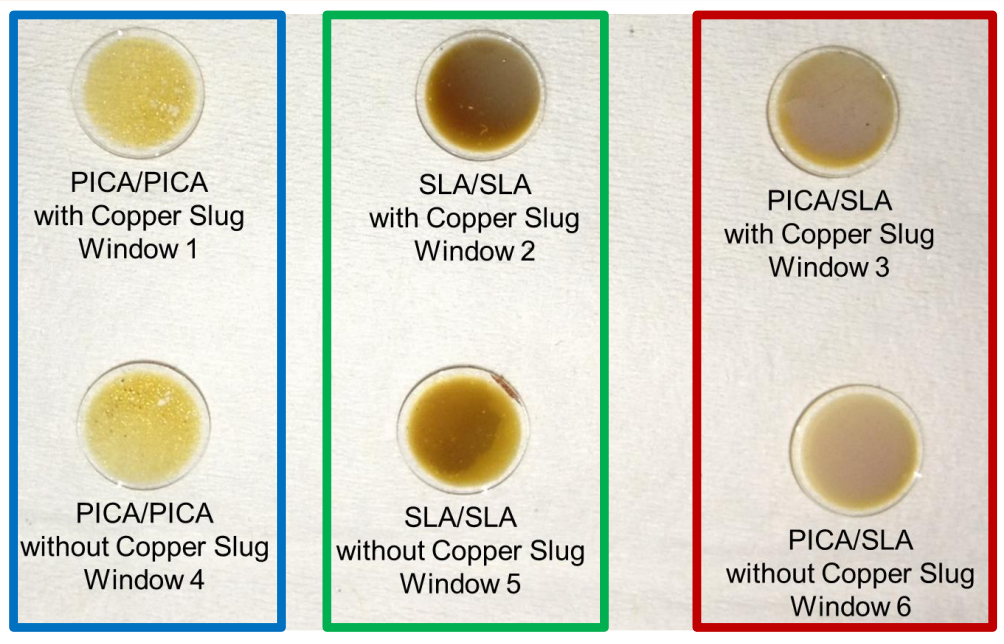

\begin{tabular}{|cccc|cc|cc|}
\hline & Initial & PICA/PICA & PICA/PICA & SLA/SLA & SLA/SLA & PICA/SLA & PICA/SLA \\
\hline $\begin{array}{c}\text { Copper } \\
\text { Slug? }\end{array}$ & --- & Yes & No & Yes & No & Yes & No \\
$\begin{array}{c}\text { Window } \\
\text { Number }\end{array}$ & --- & 1 & 4 & 2 & 5 & 3 & 6 \\
$\begin{array}{c}\text { Max S Soss } \\
\text { (\%) }\end{array}$ & $\mathbf{1 . 8}$ & $\mathbf{1 2 . 2}$ & $\mathbf{1 3 . 1}$ & $\mathbf{2 7 . 9}$ & $\mathbf{2 9 . 0}$ & $\mathbf{1 9 . 7}$ & 19.1 \\
\hline
\end{tabular}

- Signal loss of $12-30 \%$ from ablation products coating the sapphire window in both

$2.7 \mu \mathrm{m}$ and $4.3 \mu \mathrm{m}$ bands

- SLA/SLA likely an over-test

- PICA/SLA more representative with 20\% signal loss 


\section{Conclusions}

- Initial

- Transmission of radiometer window 88\% up to $5.5 \mu \mathrm{m}$

- Absorbance of heat flux sensor 95\%

- Radiometer measures about $85 \%$ of total expected radiation, which is correctable through calibration

- About $1.8 \%$ loss due to variation in wavelength sensitivity

- Post-mARC Testing

- Decreased radiometer window transmission at $~ 3 \mu \mathrm{m}, \sim 4.5 \mu \mathrm{m},>5.5 \mu \mathrm{m}$

- 12 - $30 \%$ of signal lost due TPS ablation products

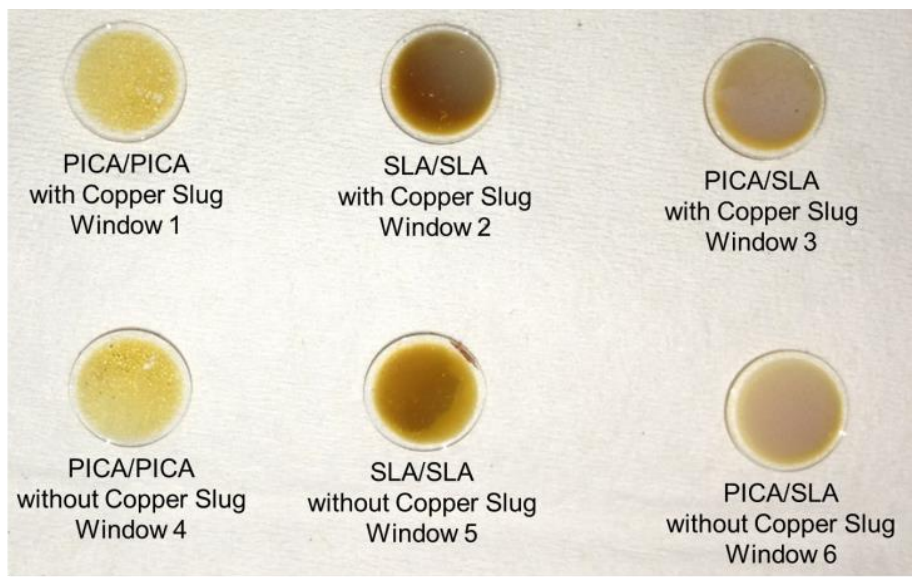




\section{Future Work}

- How "flight-like" were the conditions in the mARC?

\section{Flight Matched Conditions}

Peak heatshield heat flux on TPS \#1

Peak backshell heat flux at window location
Not Flight Matched Conditions

Heat profile

Length scale

\section{Heat load}

- Other questions:

- How does cold-soak during interplanetary transit impact window transmission and ablation product deposition?

- How does window transmission degrade as a function of time during entry?

Repeat flight and mARC test CFD

$>$ Blowing boundary condition that matches the mass loss rate predicted by material response models

$>$ Compare concentration of ablation products over the window

$>$ If the flight concentration is less than the mARC test, these results will be treated as an over-test 


\section{Thank you! Questions?}




\section{Appendix}




\section{XPS Results - Atomic \% (Average)}

\begin{tabular}{|cccc}
\hline & $\begin{array}{c}\text { PICA/PICA } \\
\text { Window 4 }\end{array}$ & $\begin{array}{c}\text { SLA/SLA } \\
\text { Window 2 }\end{array}$ & $\begin{array}{c}\text { PICA/SLA } \\
\text { Window 6 }\end{array}$ \\
\hline C 1s & $59.8 \%$ & $54.7 \%$ & $52.1 \%$ \\
\hline O 1s & $29.1 \%$ & $27.9 \%$ & $30.2 \%$ \\
\hline Si 2p & $4.5 \%$ & $12.5 \%$ & $11.1 \%$ \\
\hline N 1s & $3.7 \%$ & $3.9 \%$ & $4.8 \%$ \\
\hline Cu 2p3 & $3.0 \%$ & $1.0 \%$ & $1.9 \%$ \\
\hline
\end{tabular}

- Identified elements (carbon, oxygen, and silicon) support conclusions from FTIR data

- Windows in PICA/PICA models contained the most carbon

- Windows in SLA/SLA models contained the most silicon

- Presence of silicon in PICA/PICA windows could be from RTV-560 used to adhere the TPS to the bracket or from insulating tape used inside the chamber

- Copper is assumed to come from the mARC electrodes as the arc erodes them 